CRYSTALLOGRAPHIC COMMUNICATIONS

ISSN 2056-9890

\section{The first spontaneous resolution of a sulfoxide: Dianin's compound analogue, $(R)$-4-(4-hydroxy- phenyl)-2,2,4-trimethylthiachroman-1-oxide}

\author{
James H. Gall, ${ }^{\mathrm{a}}$ J. Derek White, ${ }^{\mathrm{a}}$ David D. MacNicol ${ }^{\mathrm{a}}$ and Christopher S. \\ Frampton $^{\mathbf{b} *}$
}

Received 12 September 2018

Accepted 11 October 2018

Edited by V. Khrustalev, Russian Academy of Sciences, Russia

Keywords: crystal structure; thia-Dianin's compound; hydrogen bonding; spontaneous resolution.

CCDC reference: 1872666

Supporting information: this article has supporting information at journals.iucr.org/e

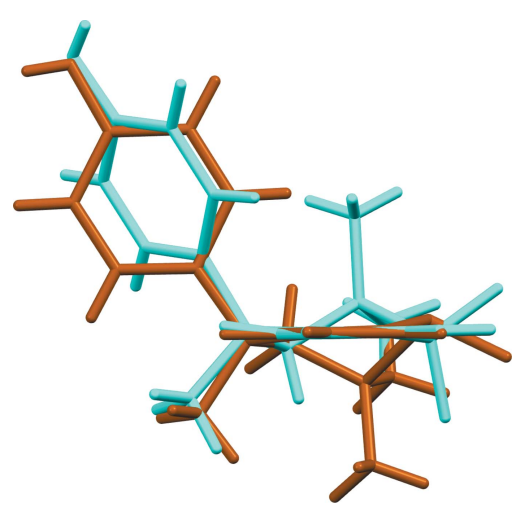

OPEN $\odot$ ACCESS

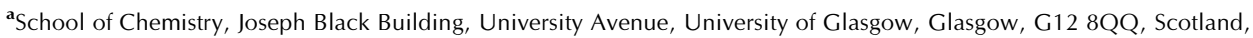
and ${ }^{\mathbf{b}}$ Experimental Techniques Centre, Brunel University London, Kingston Lane, Uxbridge, UB8 3PH, England.

${ }^{*}$ Correspondence e-mail: chris.frampton@brunel.ac.uk

The title sulfoxide, $\mathrm{C}_{18} \mathrm{H}_{20} \mathrm{O}_{2} \mathrm{~S}$, was prepared by controlled oxidation of thiaDianin's compound using hydrogen peroxide in glacial acetic acid. On recrystallization from glacial acetic acid, it was found to form unsolvated, spontaneously resolved crystals, the initial crystal structure analysis revealing the presence of both sulfoxide epimers in the crystal. On multiple recrystallization a single epimer was observed, with crystallization occurring in the unchanged orthorhombic space group $P 2_{1} 2_{1} 2_{1}$, with $Z^{\prime}=1$. The molecule possesses a distal conformation, referring to the juxtaposition of the $p$-hydoxyphenyl substituent with respect to its syn-related methyl group, with the sulfoxide oxygen atom anti to the aromatic substituent. The molecular packing features $\mathrm{O}-\mathrm{H} \cdots \mathrm{O}$ hydrogen bond chains running parallel to the $b$ axis of the unit cell.

\section{Chemical context}

A significant body of work in the literature relates to specifically targeted structural modification of Dianin's compound, 4-(4-hydroxyphenyl)-2,2,4-trimethylchroman 2, (MacNicol, 1984; Finocchiaro \& Failla, 1996; Collet \& Jacques, 1976; Frampton et al., 2017a,b,c). Crystallization of the new compounds has normally resulted in one of two outcomes: formation of clathrates in the space group $R \overline{3}$ (or $R 3$ ) or spontaneous resolution, also a subject of much current interest (Pérez-García \& Amabilino, 2007), to form an unsolvated conglomerate in space group $P 2_{1} 2_{1} 2_{1}$, with $Z^{\prime}=1$, in which the individual crystals are formed by supramolecular assembly of a single enantiomer. A notable departure from the above crystallization modes has, however, been found in the case of Dianin's sulfone 4, (Frampton et al., 1992), which crystallizes unsolvated in the polar monoclinic space group $C$ c, with $Z^{\prime}=$ 1 , and these crystals exhibited a significant SHG effect. The present work was undertaken to establish if the corresponding sulfoxide 1 would retain the clathrating ability of its immediate progenitor thia-Dianin's compound $\mathbf{3}$, or would undergo spontaneous resolution, alternative possibilities being the formation of a polar monoclinic crystal or crystallization in a more frequently encountered space group. Interestingly, the achiral bis-sulfoxide trans-( $R, S)$ - $\alpha, \alpha^{\prime}$-di-tert-butylsulfinyl-paraxylene undergoes conformational spontaneous resolution in the space group $P 22_{1} 2_{1} 2_{1}$ : on dissolution, rapid conformational racemization occurs at room temperature; however, the authors make the point that at $173 \mathrm{~K}$, from calculations, it 
could be possible to obtain one chiral conformation from a single crystal (Xu et al., 2014). Accordingly, the sulfoxide 1 was prepared by controlled oxidation of $\mathbf{3}$ as described in Section 5 , and its crystal structure determined.<smiles>CC1(C)[Z]c2ccccc2C(C)(c2ccc(O)cc2)C1</smiles>

$$
\begin{array}{ll}
1 & Z=S O \\
2 & Z=O \\
3 & Z=S \\
4 & Z=\mathrm{SO}_{2}
\end{array}
$$<smiles>CC1(C)CC(C)(c2ccc(O)cc2O)c2ccccc2O1</smiles><smiles>CC1(C)CC(C)(c2ccc(O)cc2)c2ccc3ccccc3c2S1</smiles>

\section{Structural commentary}

Initial attempts to determine the crystal structure of $\mathbf{1}$ revealed the presence of both sulfoxide epimers in the crystal in a ratio of approximately 90:10. It was found that multiple

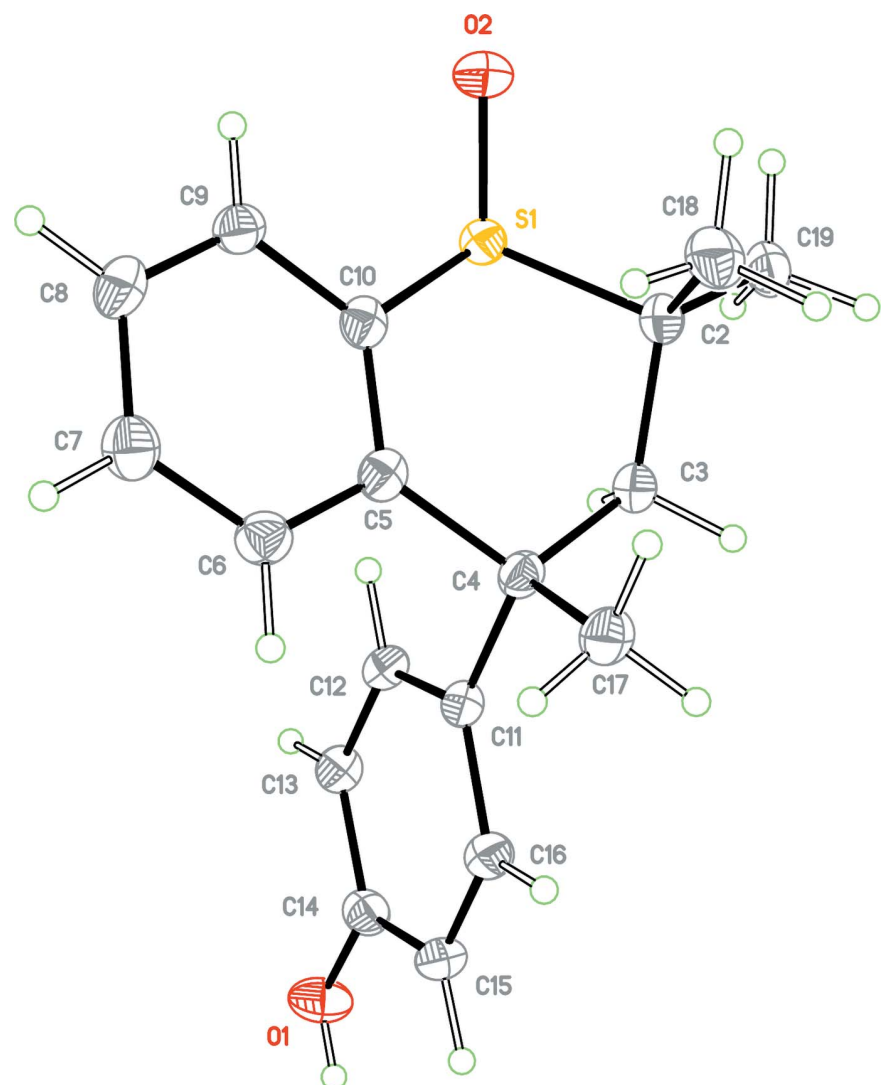

Figure 1

View of molecule $\mathbf{1}$ with the atom-labelling scheme. Ellipsoids are drawn at the $50 \%$ probability level. recrystallization of $\mathbf{1}$ from glacial acetic acid yielded a single epimer, the structure of which is presented here. The crystal structure of $\mathbf{1}$ is orthorhombic, space group $P 2_{1} 2_{1} 2_{1}$ with a single independent molecule in the asymmetric unit, $\left(Z^{\prime}=1\right)$, Fig. 1. The molecule possesses a distal conformation, this referring to the juxtaposition of the $p$-hydroxyphenyl substituent with respect to the syn-related methyl group. The $\mathrm{C} 2-$ $\mathrm{C} 3-\mathrm{C} 4-\mathrm{C} 11$ torsion angle is $154.0(2)^{\circ}$, the corresponding torsion angle for racemic Dianin's compound 2 has a magnitude of $80.67^{\circ}$ (Lee et al., 2014) and for 4-(4-hydroxyphenyl)2,2,4-trimethylchroman-1,1-dioxide 4, it is $76.8^{\circ}$ (Frampton et al., 1992). The expected torsional angle value for a distal conformation is $160^{\circ}$ whereas that for a proximal conformation is $80^{\circ}$. The torsion angle $\mathrm{S} 1-\mathrm{C} 2-\mathrm{C} 3-\mathrm{C} 4$, defining the heterocyclic ring chirality, has a value of $-67.3(2)^{\circ}$. Fig. 2 shows an overlay of $\mathbf{1}$ (brown) with sulfone $\mathbf{4}$ (cyan). In this figure, the six aromatic atoms of the chroman unit for each structure have been overlaid using the standard molecule overlay routine in Mercury (Macrae et al., 2008), resulting in an r.m.s. displacement of $0.0147 \AA$, and this clearly demonstrates the difference between the distal and proximal conformations of $\mathbf{1}$ and $\mathbf{4}$, respectively. The absolute configuration of $\mathbf{1}$, was determined as being $R$ at the chiral centre $\mathrm{C} 4$ by anomalous dispersion methods, (Parsons et al. 2013), the Flack $x$ parameter was determined as $-0.002(7)$ using 1246 quotients $\left[\left(I^{+}\right)-\left(I^{-}\right)\right] /\left[\left(I^{+}\right)+\left(I^{-}\right)\right]$.

\section{Supramolecular features}

The structure of $\mathbf{1}$ is isostructural with the enantiomerically pure amine counterparts of Dianin's and thia-Dianin's compound, (R)-4-(4-aminophenyl)-2,2,4-trimethylchroman and (S)-4-(4-aminophenyl)-2,2,4-trimethylthiachroman, both

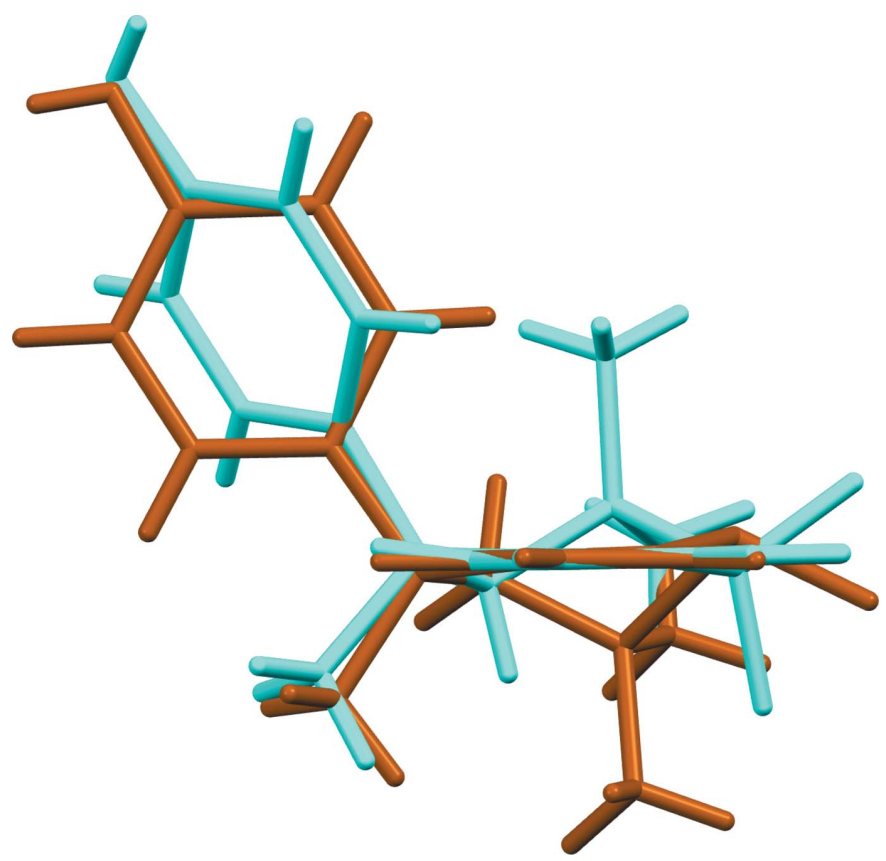

Figure 2

View of the structure overlay of $\mathbf{1}$ (brown) and $\mathbf{4}$ (cyan). 


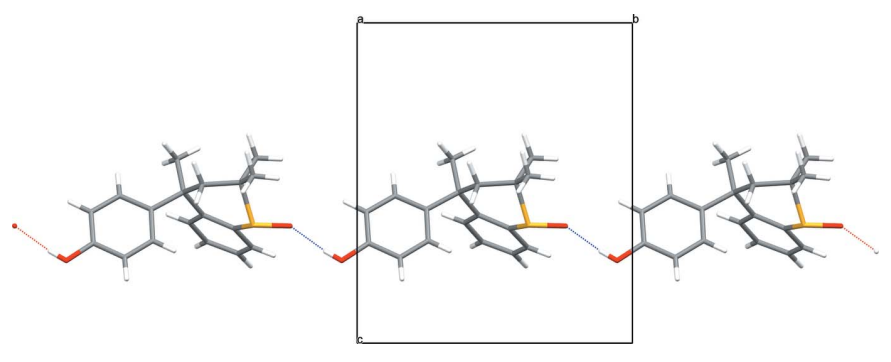

Figure 3

A partial view of the crystal packing down the $a$ axis showing the hydrogen-bonded chain. The intermolecular $\mathrm{O}-\mathrm{H} \cdots \mathrm{O}$ hydrogen bond is shown as a dotted line.

of which were obtained by spontaneous resolution (Frampton et al., 2011), and also surprisingly isostructural with the enantiomerically pure forms of 4-(4-hydroxyphenyl)-2,2,4trimethylchroman, 2 (Lloyd \& Bredenkamp, 2005) and 4-(2,4dihydroxyphenyl)-2,2,4-trimethylchroman, 5 (Beresford et al., 1999). The crystal packing is dominated by the formation of an extended linear hydroxy - $\mathrm{OH}$ to sulfoxide $\mathrm{O}$, hydrogenbonded $\mathrm{O}-\mathrm{H} \cdots \mathrm{O}$ chain along the [010] direction of the unit cell, Figs. 3 and 4, Table 1.

\section{Database survey}

A search of the Cambridge Structural Database (CSD, Version 5.39 update August 2018; Groom et al., 2016) for the thia-Dianin's framework, 3, yielded 15 hits, all of which were genuine examples of analogues of the material under investigation. Although there are no entries for the empty racemic $R \overline{3}$ host of thia-Dianin's compound, there are seven entries for

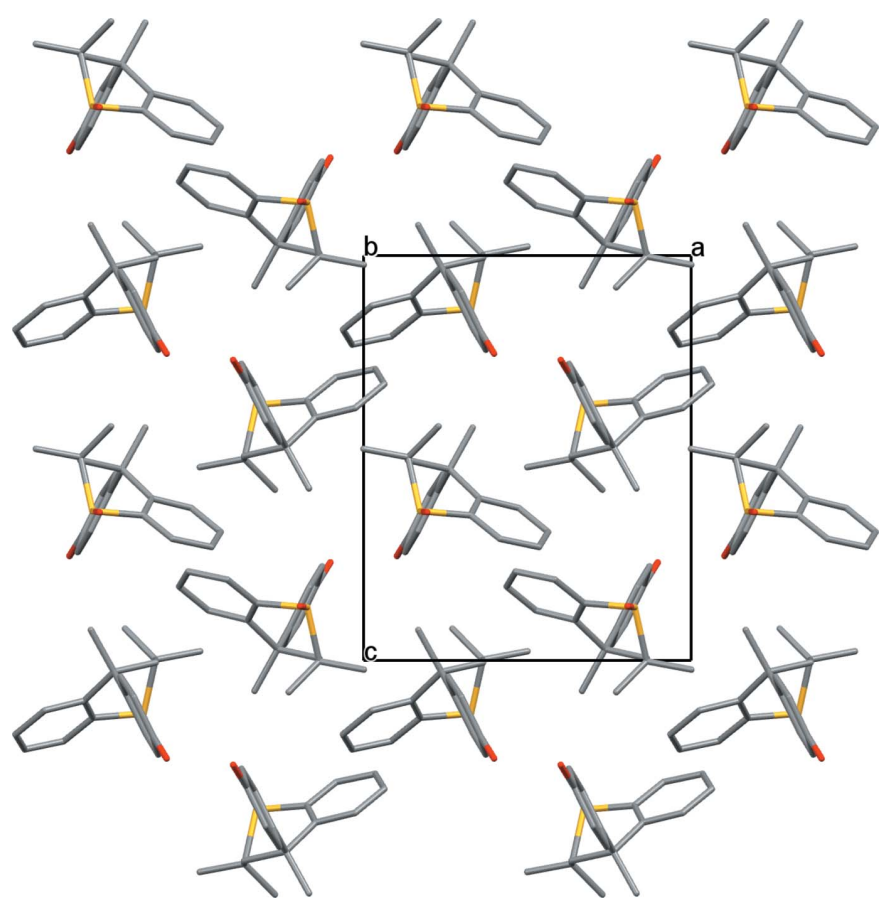

Figure 4

View of the crystal packing of $\mathbf{1}$ down the $b$ axis.
Table 1

Hydrogen-bond geometry $\left(\AA{ }^{\circ}{ }^{\circ}\right)$.

\begin{tabular}{lllll}
\hline$D-\mathrm{H} \cdots A$ & $D-\mathrm{H}$ & $\mathrm{H} \cdots A$ & $D \cdots A$ & $D-\mathrm{H} \cdots A$ \\
\hline $\mathrm{O} 1-\mathrm{H} 1 \cdots \mathrm{O} 2^{\mathrm{i}}$ & $0.77(3)$ & $1.92(3)$ & $2.685(3)$ & $169(3)$ \\
\hline
\end{tabular}

Symmetry code: (i) $x, y-1, z$.

the following host-guest clathrates: ethanol (HPTHCR; MacNicol et al., 1969), 2,5,5-trimethylhex-3-yn-2-ol (TCHHXO; MacNicol \& Wilson, 1971), cyclopentane (METCCP; Hardy et al., 1979) and isopropanol at four different temperatures demonstrating three commensurate phase changes in the host lattice (VANFOI, $371 \mathrm{~K}$, VANFOI01, $295 \mathrm{~K}$, VANFOI02, $200 \mathrm{~K}$ and VANFUO, $90 \mathrm{~K}$; Frampton et al., 2017a). Thia-Dianin's compound, 3, was also found in the 1:1 quasi-racemic $R 3$ host with Dianin's compound, 2, in the following three entries: apohost (BIBNAD and BIBNAD01), $\mathrm{CCl}_{4} / \mathrm{H}_{2} \mathrm{O}$ host-guest clathrate (HIDQAO) (Frampton et al., 2013). The structure and absolute stereochemistry determination of the resolved $S$-enantiomer of thia-Dianin's compound used in the formation of the quasi-racemates above (BIBNEH: Frampton et al., 2013). Four further examples demonstrating a slightly modified framework include the 6-methyl analogue (HPMTCM; Hardy et al., 1977), the cycloctane host-guest clathrate of the 8-methyl analogue (MSOCYO10; Hardy et al., 1979), the oxidized sulfone, 4, (KUTDUY; Frampton et al., 1992) and 4-(4-hydroxyphenyl)-2,2,4-trimethyl-7,8-benzothiachroman $\mathbf{6}$, a fused-ring counterpart of thia-Dianin's compound (JELROK; Frampton et al., 2017c).

\section{Synthesis and crystallization}

Preparation of 1: 4-(4-hydroxyphenyl)-2,2,4-trimethylthiachroman 3 (MacNicol, 1969) (0.25 g, $0.88 \mathrm{mmol}$ ) was dissolved in glacial acetic acid $(10 \mathrm{~mL})$ and a $50 \%$ excess of $30 \%$ hydrogen peroxide $(0.15 \mathrm{~mL}, 1.32 \mathrm{mmol})$ added. After the reaction was left overnight at $c a 278 \mathrm{~K}$, the precipitated white solid was filtered off, washed several times with ether, and initially recrystallized from aqueous dimethyl sulfoxide yielding $0.168 \mathrm{~g},(63 \%)$ of product. A further recrystallization from glacial acetic acid gave colourless crystals which were analysed by X-ray diffraction as described in the text. The crystals were obtained by spontaneous resolution on crystallization, yielding a 50:50 mixture of the pure enantiomers. These crystals also incorporated both spontaneously resolved sulfoxide epimers, four further recrystallizations were performed giving a single epimer of purity greater than $99 \%$ $\left[500 \mathrm{MHz}{ }^{1} \mathrm{H}\right.$ NMR, DMSO- $d_{6}$ solution analysis gave 99.5 (2)\% purity] and the very minor residual second epimer was undetectable in the subsequent X-ray analysis. These crystals melted over a wide range, ca 513-536 K, possibly arising from sulfoxide epimerization, along with decomposition, at high temperature. MS $\left[\mathrm{EI}^{+}\right]$: 300.1178, $\mathrm{C}_{18} \mathrm{H}_{20} \mathrm{O}_{2} \mathrm{~S}$, calculated 300.1184; ${ }^{1} \mathrm{H}$ NMR $\left(400 \mathrm{MHz}, \mathrm{DMSO}-d_{6}\right): \delta 0.94(s$, $3 \mathrm{H}), 1.31(s, 3 \mathrm{H}), 1.67(s, 3 \mathrm{H}), 2.26\left(q, 2 \mathrm{H}, \delta_{\mathrm{AB}}=0.45 \mathrm{ppm}, J_{\mathrm{AB}}\right.$ 
= 15.1 Hz), 6.6-7.7 (aromatic, 8H), 9.27(s, 1H); FT-IR ( $v_{\max }$, ATR, $\left.\mathrm{cm}^{-1}\right): 3176(b r), 3197$ (minor) $[v(\mathrm{O}-\mathrm{H})] ; 1017[v(\mathrm{~S}-$ $\mathrm{O})$ ].

\section{Refinement}

Crystal data, data collection and structure refinement details are summarized in Table 2.

The hydrogen atom of the $\mathrm{OH}$ group was localized in the difference-Fourier map and refined isotropically. The other hydrogen atoms were placed in calculated positions and refined within the riding model with $\mathrm{C}-\mathrm{H}=0.95-0.99 \AA$ and fixed isotropic displacement parameters $\left[U_{\text {iso }}(\mathrm{H})=1.5 U_{\text {eq }}(\mathrm{C})\right.$ for the methyl groups and $1.2 U_{\mathrm{eq}}(\mathrm{C})$ for the other groups].

\section{References}

Beresford, T. W., Frampton, C. S., Gall, J. H. \& MacNicol, D. D. (1999). Zh. Strukt. Khim. 40, 872-882.

Collet, A. \& Jacques, J. (1976). Isr. J. Chem. 15, 82-83.

Finocchiaro, P. \& Failla, S. (1996). In Comprehensive Supramolecular Chemistry, Vol. 6, edited by D. D. MacNicol, F. Toda \& R. Bishop, ch. 18, pp. 618-627. Oxford: Elsevier.

Frampton, C. S., Gall, J. H. \& MacNicol, D. D. (2017b). CrystEngComm, 19, 5703-5706.

Frampton, C. S., Ketuly, K. K., Ali, H. B. M., Azizan, A. H. S., Gall, J. H. \& MacNicol, D. D. (2017a). CrystEngComm, 19, 2653-2659.

Frampton, C. S., Ketuly, K. A., Hadi, A., Gall, J. H. \& MacNicol, D. D. (2013). Chem. Commun. 49, 7198-7200.

Frampton, C. S., MacNicol, D. D., Mallinson, P. R. \& White, J. D. (1992). J. Crystallogr. Spectrosc. Res. 22, 551-555.

Frampton, C. S., MacNicol, D. D. \& Wilson, D. R. (2011). Acta Cryst. C67, o188-o191.

Frampton, C. S., McKendrick, J. J. \& MacNicol, D. D. (2017c). Acta Cryst. E73, 1662-1665.

Groom, C. R., Bruno, I. J., Lightfoot, M. P. \& Ward, S. C. (2016). Acta Cryst. B72, 171-179.

Hardy, A. D. U., McKendrick, J. J. \& MacNicol, D. D. (1977). J. Chem. Soc. Perkin Trans. 2, pp. 1145-1147.

Hardy, A. D. U., McKendrick, J. J. \& MacNicol, D. D. (1979). J. Chem. Soc. Perkin Trans. 2, pp. 1072-1077.

Lee, J. J., Sobolev, A. N., Turner, M. J., Fuller, R. O., Iversen, B. B., Koutsantonis, G. A. \& Spackman, M. A. (2014). Cryst. Growth Des. 14, 1296-1306.

Lloyd, G. O. \& Bredenkamp, M. W. (2005). Acta Cryst. E61, o1512o1514.

MacNicol, D. D. (1969). J. Chem. Soc. D, p. 836.

MacNicol, D. D. (1984). Inclusion Compounds, Vol. 2, edited by J. L. Atwood, J. E. D. Davies \& D. D. MacNicol, ch. 1, pp. 12-32. New York: Academic Press.

MacNicol, D. D., Mills, H. H. \& Wilson, F. B. (1969). J. Chem. Soc. D, pp. 1332-1333.

MacNicol, D. D. \& Wilson, F. B. (1971). J. Chem. Soc. D, pp. 786-787.
Table 2

Experimental details.

\begin{tabular}{|c|c|}
\hline \multicolumn{2}{|l|}{ Crystal data } \\
\hline Chemical formula & $\mathrm{C}_{18} \mathrm{H}_{20} \mathrm{O}_{2} \mathrm{~S}$ \\
\hline$M_{\mathrm{r}}$ & 300.40 \\
\hline Crystal system, space group & Orthorhombic, $P 2_{1} 2_{1} 2_{1}$ \\
\hline Temperature (K) & 100 \\
\hline$a, b, c(\AA)$ & $\begin{array}{l}10.4311(3), 11.0892(3), \\
\quad 12.8868(3)\end{array}$ \\
\hline$V\left(\AA^{3}\right)$ & $1490.65(7)$ \\
\hline$Z$ & 4 \\
\hline Radiation type & $\mathrm{Cu} K \alpha$ \\
\hline$\mu\left(\mathrm{mm}^{-1}\right)$ & 1.94 \\
\hline Crystal size $(\mathrm{mm})$ & $0.18 \times 0.12 \times 0.10$ \\
\hline \multicolumn{2}{|l|}{ Data collection } \\
\hline Diffractometer & $\begin{array}{l}\text { Rigaku Oxford Diffraction Super- } \\
\text { Nova, Dualflex, AtlasS2 }\end{array}$ \\
\hline Absorption correction & $\begin{array}{l}\text { Gaussian (CrysAlis PRO; Rigaku } \\
\text { OD, 2015) }\end{array}$ \\
\hline$T_{\min }, T_{\max }$ & $0.786,0.844$ \\
\hline $\begin{array}{l}\text { No. of measured, independent and } \\
\text { observed }[I>2 \sigma(I)] \text { reflections }\end{array}$ & $5735,3046,2982$ \\
\hline$R_{\text {int }}$ & 0.016 \\
\hline$(\sin \theta / \lambda)_{\max }\left(\AA^{-1}\right)$ & 0.625 \\
\hline \multicolumn{2}{|l|}{ Refinement } \\
\hline$R\left[F^{2}>2 \sigma\left(F^{2}\right)\right], w R\left(F^{2}\right), S$ & $0.028,0.075,1.03$ \\
\hline No. of reflections & 3046 \\
\hline No. of parameters & 197 \\
\hline $\mathrm{H}$-atom treatment & $\begin{array}{l}\mathrm{H} \text { atoms treated by a mixture of } \\
\text { independent and constrained } \\
\text { refinement }\end{array}$ \\
\hline$\Delta \rho_{\max }, \Delta \rho_{\min }\left(\mathrm{e} \AA^{-3}\right)$ & $0.27,-0.29$ \\
\hline Absolute structure & $\begin{array}{l}\text { Flack x determined using } 1246 \\
\quad \text { quotients }\left[\left(I^{+}\right)-\left(I^{-}\right)\right] /\left[\left(I^{+}\right)+\left(I^{-}\right)\right] \\
\quad \text { (Parsons } \text { et al. } 2013)\end{array}$ \\
\hline Absolute structure parameter & $-0.002(7)$ \\
\hline
\end{tabular}

Computer programs: CrysAlis PRO (Rigaku OD, 2015), SHELXD2014/6 (Schneider \& Sheldrick, 2002), SHELXL2014/6 (Sheldrick, 2015), SHELXTL (Sheldrick, 2008), Mercury (Macrae et al., 2008) and publCIF (Westrip, 2010).

Macrae, C. F., Bruno, I. J., Chisholm, J. A., Edgington, P. R., McCabe, P., Pidcock, E., Rodriguez-Monge, L., Taylor, R., van de Streek, J. \& Wood, P. A. (2008). J. Appl. Cryst. 41, 466-470.

Parsons, S., Flack, H. D. \& Wagner, T. (2013). Acta Cryst. B69, 249 259.

Pérez-García, L. \& Amabilino, D. B. (2007). Chem. Soc. Rev. 36, 941967.

Rigaku OD (2015). CrysAlis PRO. Rigaku Oxford Diffraction, Yarnton, England.

Schneider, T. R. \& Sheldrick, G. M. (2002). Acta Cryst. D58, 17721779.

Sheldrick, G. M. (2008). Acta Cryst. A64, 112-122.

Sheldrick, G. M. (2015). Acta Cryst. C71, 3-8.

Westrip, S. P. (2010). J. Appl. Cryst. 43, 920-925.

Xu, Z., Liu, H., Mahmood, M. H. R., Cai, Y., Xu, X. \& Tang, Y. (2014). CrystEngComm, 16, 3839-3842. 


\section{supporting information}

Acta Cryst. (2018). E74, 1633-1636 [https://doi.org/10.1107/S2056989018014366]

The first spontaneous resolution of a sulfoxide: Dianin's compound analogue, $(R)$-4-(4-hydroxyphenyl)-2,2,4-trimethylthiachroman-1-oxide

James H. Gall, J. Derek White, David D. MacNicol and Christopher S. Frampton

Computing details

Data collection: CrysAlis PRO (Rigaku OD, 2015); cell refinement: CrysAlis PRO (Rigaku OD, 2015); data reduction: CrysAlis PRO (Rigaku OD, 2015); program(s) used to solve structure: SHELXD2014/6 (Schneider \& Sheldrick, 2002); program(s) used to refine structure: SHELXL2014/6 (Sheldrick, 2015); molecular graphics: SHELXTL (Sheldrick, 2008), Mercury (Macrae et al., 2008); software used to prepare material for publication: SHELXTL (Sheldrick, 2008), Mercury (Macrae et al., 2008) and publCIF (Westrip, 2010).

(R)-4-(4-Hydroxyphenyl)-2,2,4-trimethyl-3H-1 $\lambda^{4}$-benzothiopyran-1-one

Crystal data

$\mathrm{C}_{18} \mathrm{H}_{20} \mathrm{O}_{2} \mathrm{~S}$

$M_{r}=300.40$

Orthorhombic, $P 2_{1} 2_{1} 2_{1}$

$a=10.4311(3) \AA$

$b=11.0892(3) \AA$

$c=12.8868(3) \AA$

$V=1490.65(7) \AA^{3}$

$Z=4$

$F(000)=640$

\section{Data collection}

Rigaku Oxford Diffraction SuperNova, Dualflex, AtlasS2

diffractometer

Radiation source: fine-focus sealed X-ray tube, Enhance $(\mathrm{Cu}) \mathrm{X}$-ray Source

Detector resolution: 5.2921 pixels $\mathrm{mm}^{-1}$ $\omega$ scans

Absorption correction: gaussian

(CrysAlisPro; Rigaku OD, 2015)

\section{Refinement}

Refinement on $F^{2}$

Least-squares matrix: full

$R\left[F^{2}>2 \sigma\left(F^{2}\right)\right]=0.028$

$w R\left(F^{2}\right)=0.075$

$S=1.03$

3046 reflections

197 parameters

0 restraints
$D_{\mathrm{x}}=1.339 \mathrm{Mg} \mathrm{m}^{-3}$

$\mathrm{Cu} K \alpha$ radiation, $\lambda=1.54184 \AA$

Cell parameters from 3948 reflections

$\theta=5.3-74.2^{\circ}$

$\mu=1.94 \mathrm{~mm}^{-1}$

$T=100 \mathrm{~K}$

Block, colourless

$0.18 \times 0.12 \times 0.10 \mathrm{~mm}$

$T_{\min }=0.786, T_{\max }=0.844$

5735 measured reflections

3046 independent reflections

2982 reflections with $I>2 \sigma(I)$

$R_{\text {int }}=0.016$

$\theta_{\text {max }}=74.5^{\circ}, \theta_{\text {min }}=5.3^{\circ}$

$h=-12 \rightarrow 13$

$k=-12 \rightarrow 13$

$l=-16 \rightarrow 13$

Primary atom site location: difference Fourier map

Secondary atom site location: difference Fourier map

Hydrogen site location: mixed

$\mathrm{H}$ atoms treated by a mixture of independent and constrained refinement 
$w=1 /\left[\sigma^{2}\left(F_{\mathrm{o}}^{2}\right)+(0.0407 P)^{2}+0.434 P\right]$

where $P=\left(F_{\mathrm{o}}^{2}+2 F_{\mathrm{c}}^{2}\right) / 3$

$(\Delta / \sigma)_{\max }=0.001$

$\Delta \rho_{\max }=0.27 \mathrm{e} \AA^{-3}$

$\Delta \rho_{\min }=-0.29$ e $\AA^{-3}$
Absolute structure: Flack x determined using 1246 quotients $\left[\left(I^{+}\right)-\left(I^{-}\right)\right] /\left[\left(I^{+}\right)+\left(I^{-}\right)\right]$(Parsons et al., 2013)

Absolute structure parameter: -0.002 (7)

\section{Special details}

Geometry. All esds (except the esd in the dihedral angle between two 1.s. planes) are estimated using the full covariance matrix. The cell esds are taken into account individually in the estimation of esds in distances, angles and torsion angles; correlations between esds in cell parameters are only used when they are defined by crystal symmetry. An approximate (isotropic) treatment of cell esds is used for estimating esds involving l.s. planes.

Fractional atomic coordinates and isotropic or equivalent isotropic displacement parameters $\left(\AA^{2}\right)$

\begin{tabular}{|c|c|c|c|c|}
\hline & $x$ & $y$ & $z$ & $U_{\text {iso }} * / U_{\text {eq }}$ \\
\hline S1 & $0.17202(5)$ & $0.62246(5)$ & $0.63136(4)$ & $0.01942(13)$ \\
\hline $\mathrm{O} 1$ & $0.10168(17)$ & $-0.05587(16)$ & $0.74216(15)$ & $0.0286(4)$ \\
\hline H1 & $0.120(3)$ & $-0.115(3)$ & $0.713(2)$ & $0.031(8)^{*}$ \\
\hline $\mathrm{O} 2$ & $0.19457(17)$ & $0.75661(14)$ & $0.63392(14)$ & $0.0290(4)$ \\
\hline $\mathrm{C} 2$ & $0.1359(2)$ & $0.5775(2)$ & $0.49805(16)$ & $0.0213(5)$ \\
\hline $\mathrm{C} 3$ & $0.1350(2)$ & $0.4390(2)$ & $0.50523(16)$ & $0.0203(4)$ \\
\hline $\mathrm{H} 3 \mathrm{~A}$ & 0.1008 & 0.4077 & 0.4388 & $0.024 *$ \\
\hline $\mathrm{H} 3 \mathrm{~B}$ & 0.0729 & 0.4164 & 0.5600 & $0.024 *$ \\
\hline $\mathrm{C} 4$ & $0.2625(2)$ & $0.3703(2)$ & $0.52807(15)$ & $0.0183(4)$ \\
\hline $\mathrm{C} 5$ & $0.3535(2)$ & $0.4405(2)$ & $0.59971(15)$ & $0.0191(4)$ \\
\hline C6 & $0.4737(2)$ & $0.3919(2)$ & $0.62341(17)$ & $0.0229(4)$ \\
\hline H6 & 0.4947 & 0.3139 & 0.5981 & $0.027^{*}$ \\
\hline $\mathrm{C} 7$ & $0.5633(2)$ & $0.4536(2)$ & $0.68256(18)$ & $0.0261(5)$ \\
\hline H7 & 0.6437 & 0.4175 & 0.6977 & $0.031^{*}$ \\
\hline $\mathrm{C} 8$ & $0.5354(2)$ & $0.5688(2)$ & $0.71980(18)$ & $0.0246(5)$ \\
\hline $\mathrm{H} 8$ & 0.5973 & 0.6126 & 0.7585 & $0.030^{*}$ \\
\hline $\mathrm{C} 9$ & $0.4163(2)$ & $0.6183(2)$ & $0.69968(15)$ & $0.0211(4)$ \\
\hline H9 & 0.3956 & 0.6962 & 0.7255 & $0.025^{*}$ \\
\hline $\mathrm{C} 10$ & $0.3267(2)$ & $0.55413(19)$ & $0.64149(15)$ & $0.0190(4)$ \\
\hline C11 & $0.2254(2)$ & $0.2522(2)$ & $0.58256(16)$ & $0.0178(4)$ \\
\hline $\mathrm{C} 12$ & $0.1659(2)$ & $0.25810(19)$ & $0.67999(16)$ & $0.0195(4)$ \\
\hline H12 & 0.1519 & 0.3348 & 0.7109 & $0.023^{*}$ \\
\hline $\mathrm{C} 13$ & $0.1269(2)$ & $0.1558(2)$ & $0.73240(16)$ & $0.0201(4)$ \\
\hline H13 & 0.0890 & 0.1629 & 0.7991 & $0.024^{*}$ \\
\hline C14 & $0.1431(2)$ & $0.04203(19)$ & $0.68765(17)$ & $0.0206(4)$ \\
\hline $\mathrm{C} 15$ & $0.2012(2)$ & $0.0339(2)$ & $0.59057(18)$ & $0.0224(4)$ \\
\hline H15 & 0.2127 & -0.0427 & 0.5589 & $0.027^{*}$ \\
\hline $\mathrm{C} 16$ & $0.2426(2)$ & $0.1379(2)$ & $0.53978(16)$ & 0.0207 (4) \\
\hline H16 & 0.2836 & 0.1306 & 0.4743 & $0.025^{*}$ \\
\hline $\mathrm{C} 17$ & $0.3331(2)$ & $0.3456(2)$ & $0.42483(16)$ & 0.0237 (4) \\
\hline H17A & 0.4110 & 0.2988 & 0.4386 & $0.036^{*}$ \\
\hline H17B & 0.3563 & 0.4224 & 0.3922 & $0.036^{*}$ \\
\hline $\mathrm{H} 17 \mathrm{C}$ & 0.2769 & 0.3000 & 0.3782 & $0.036^{*}$ \\
\hline C18 & $0.2289(2)$ & $0.6345(2)$ & $0.42175(17)$ & $0.0290(5)$ \\
\hline
\end{tabular}




$\begin{array}{lllll}\text { H18A } & 0.2208 & 0.7225 & 0.4249 & 0.044^{*} \\ \text { H18B } & 0.2091 & 0.6067 & 0.3514 & 0.044^{*} \\ \text { H18C } & 0.3167 & 0.6112 & 0.4398 & 0.044^{*} \\ \text { C19 } & -0.0004(2) & 0.6226(2) & 0.47748(17) & 0.0261(5) \\ \text { H19A } & -0.0595 & 0.5856 & 0.5273 & 0.039^{*} \\ \text { H19B } & -0.0261 & 0.6006 & 0.4068 & 0.039^{*} \\ \text { H19C } & -0.0030 & 0.7105 & 0.4851 & 0.039^{*}\end{array}$

Atomic displacement parameters $\left(\AA^{2}\right)$

\begin{tabular}{lllllll}
\hline & $U^{11}$ & $U^{22}$ & $U^{33}$ & $U^{12}$ & $U^{13}$ & $U^{23}$ \\
\hline S1 & $0.0224(2)$ & $0.0180(2)$ & $0.0179(2)$ & $0.0006(2)$ & $-0.00050(19)$ & $-0.00088(18)$ \\
O1 & $0.0335(9)$ & $0.0175(8)$ & $0.0347(9)$ & $-0.0018(7)$ & $0.0080(7)$ & $0.0026(7)$ \\
O2 & $0.0354(9)$ & $0.0181(7)$ & $0.0335(9)$ & $0.0019(7)$ & $-0.0041(8)$ & $-0.0025(7)$ \\
C2 & $0.0268(11)$ & $0.0212(11)$ & $0.0159(9)$ & $0.0007(8)$ & $-0.0021(8)$ & $0.0008(8)$ \\
C3 & $0.0235(10)$ & $0.0205(10)$ & $0.0169(9)$ & $0.0001(9)$ & $-0.0028(8)$ & $0.0003(8)$ \\
C4 & $0.0214(9)$ & $0.0186(10)$ & $0.0150(9)$ & $-0.0011(9)$ & $0.0001(7)$ & $-0.0018(9)$ \\
C5 & $0.0210(10)$ & $0.0217(10)$ & $0.0146(8)$ & $-0.0036(8)$ & $0.0026(7)$ & $0.0013(8)$ \\
C6 & $0.0229(10)$ & $0.0221(10)$ & $0.0238(10)$ & $0.0015(8)$ & $0.0011(8)$ & $-0.0022(9)$ \\
C7 & $0.0215(10)$ & $0.0308(12)$ & $0.0261(11)$ & $0.0015(9)$ & $0.0001(9)$ & $0.0005(10)$ \\
C8 & $0.0215(10)$ & $0.0292(12)$ & $0.0231(10)$ & $-0.0053(9)$ & $-0.0024(8)$ & $-0.0021(9)$ \\
C9 & $0.0263(10)$ & $0.0194(10)$ & $0.0177(9)$ & $-0.0020(9)$ & $0.0006(8)$ & $0.0000(8)$ \\
C10 & $0.0200(9)$ & $0.0218(9)$ & $0.0151(8)$ & $-0.0032(9)$ & $0.0014(9)$ & $0.0028(7)$ \\
C11 & $0.0183(9)$ & $0.0186(10)$ & $0.0166(9)$ & $0.0001(8)$ & $-0.0020(8)$ & $-0.0009(8)$ \\
C12 & $0.0202(9)$ & $0.0188(9)$ & $0.0194(9)$ & $-0.0016(9)$ & $-0.0008(9)$ & $-0.0034(8)$ \\
C13 & $0.0199(9)$ & $0.0217(11)$ & $0.0188(9)$ & $-0.0009(8)$ & $0.0019(8)$ & $-0.0003(8)$ \\
C14 & $0.0187(10)$ & $0.0184(10)$ & $0.0247(10)$ & $-0.0017(8)$ & $-0.0017(8)$ & $0.0023(9)$ \\
C15 & $0.0258(11)$ & $0.0168(10)$ & $0.0246(10)$ & $0.0005(8)$ & $-0.0016(9)$ & $-0.0039(9)$ \\
C16 & $0.0236(10)$ & $0.0193(10)$ & $0.0191(9)$ & $0.0006(9)$ & $-0.0001(8)$ & $-0.0016(9)$ \\
C17 & $0.0296(11)$ & $0.0253(11)$ & $0.0161(9)$ & $0.0002(9)$ & $0.0033(9)$ & $-0.0008(7)$ \\
C18 & $0.0387(13)$ & $0.0280(12)$ & $0.0204(10)$ & $-0.0003(11)$ & $0.0040(10)$ & $0.0048(10)$ \\
C19 & $0.0291(11)$ & $0.0270(12)$ & $0.0220(10)$ & $0.0052(11)$ & $-0.0055(8)$ & $0.0010(10)$
\end{tabular}

Geometric parameters (A, $\left.{ }^{o}\right)$

\begin{tabular}{llll}
\hline $\mathrm{S} 1-\mathrm{O} 2$ & $1.5064(16)$ & $\mathrm{C} 9-\mathrm{C} 10$ & $1.394(3)$ \\
$\mathrm{S} 1-\mathrm{C} 10$ & $1.788(2)$ & $\mathrm{C} 9-\mathrm{H} 9$ & 0.9500 \\
$\mathrm{~S} 1-\mathrm{C} 2$ & $1.828(2)$ & $\mathrm{C} 11-\mathrm{C} 16$ & $1.394(3)$ \\
$\mathrm{O} 1-\mathrm{C} 14$ & $1.363(3)$ & $\mathrm{C} 11-\mathrm{C} 12$ & $1.402(3)$ \\
$\mathrm{O} 1-\mathrm{H} 1$ & $\mathrm{C} 12-\mathrm{C} 13$ & $1.381(3)$ \\
$\mathrm{C} 2-\mathrm{C} 18$ & $0.77(3)$ & $\mathrm{C} 12-\mathrm{H} 12$ & 0.9500 \\
$\mathrm{C} 2-\mathrm{C} 19$ & $1.519(3)$ & $\mathrm{C} 13-\mathrm{C} 14$ & $1.398(3)$ \\
$\mathrm{C} 2-\mathrm{C} 3$ & $1.530(3)$ & $\mathrm{C} 13-\mathrm{H} 13$ & 0.9500 \\
$\mathrm{C} 3-\mathrm{C} 4$ & $1.539(3)$ & $\mathrm{C} 14-\mathrm{C} 15$ & $1.393(3)$ \\
$\mathrm{C} 3-\mathrm{H} 3 \mathrm{~A}$ & $1.560(3)$ & $\mathrm{C} 15-\mathrm{C} 16$ & $1.395(3)$ \\
$\mathrm{C} 3-\mathrm{H} 3 \mathrm{~B}$ & 0.9900 & $\mathrm{C} 15-\mathrm{H} 15$ & 0.9500 \\
$\mathrm{C} 4-\mathrm{C} 11$ & 0.9900 & $\mathrm{C} 16-\mathrm{H} 16$ & 0.9500 \\
$\mathrm{C} 4-\mathrm{C} 5$ & $1.535(3)$ & $\mathrm{C} 17-\mathrm{H} 17 \mathrm{~A}$ & 0.9800
\end{tabular}




\begin{tabular}{|c|c|c|c|}
\hline $\mathrm{C} 4-\mathrm{C} 17$ & $1.545(3)$ & C17-H17B & 0.9800 \\
\hline $\mathrm{C} 5-\mathrm{C} 10$ & $1.398(3)$ & $\mathrm{C} 17-\mathrm{H} 17 \mathrm{C}$ & 0.9800 \\
\hline $\mathrm{C} 5-\mathrm{C} 6$ & $1.399(3)$ & C18-H18A & 0.9800 \\
\hline $\mathrm{C} 6-\mathrm{C} 7$ & $1.387(3)$ & C18-H18B & 0.9800 \\
\hline $\mathrm{C} 6-\mathrm{H} 6$ & 0.9500 & $\mathrm{C} 18-\mathrm{H} 18 \mathrm{C}$ & 0.9800 \\
\hline $\mathrm{C} 7-\mathrm{C} 8$ & $1.395(4)$ & C19-H19A & 0.9800 \\
\hline $\mathrm{C} 7-\mathrm{H} 7$ & 0.9500 & C19-H19B & 0.9800 \\
\hline $\mathrm{C} 8-\mathrm{C} 9$ & $1.383(3)$ & $\mathrm{C} 19-\mathrm{H} 19 \mathrm{C}$ & 0.9800 \\
\hline $\mathrm{C} 8-\mathrm{H} 8$ & 0.9500 & & \\
\hline $\mathrm{O} 2-\mathrm{S} 1-\mathrm{C} 10$ & $106.02(10)$ & $\mathrm{C} 9-\mathrm{C} 10-\mathrm{S} 1$ & $115.34(17)$ \\
\hline $\mathrm{O} 2-\mathrm{S} 1-\mathrm{C} 2$ & $108.79(10)$ & $\mathrm{C} 5-\mathrm{C} 10-\mathrm{S} 1$ & $122.27(16)$ \\
\hline $\mathrm{C} 10-\mathrm{S} 1-\mathrm{C} 2$ & $98.01(10)$ & $\mathrm{C} 16-\mathrm{C} 11-\mathrm{C} 12$ & $117.0(2)$ \\
\hline $\mathrm{C} 14-\mathrm{O} 1-\mathrm{H} 1$ & $110(2)$ & $\mathrm{C} 16-\mathrm{C} 11-\mathrm{C} 4$ & 124.22 (19) \\
\hline $\mathrm{C} 18-\mathrm{C} 2-\mathrm{C} 19$ & $110.19(19)$ & $\mathrm{C} 12-\mathrm{C} 11-\mathrm{C} 4$ & $118.76(19)$ \\
\hline $\mathrm{C} 18-\mathrm{C} 2-\mathrm{C} 3$ & $117.26(19)$ & $\mathrm{C} 13-\mathrm{C} 12-\mathrm{C} 11$ & $122.0(2)$ \\
\hline $\mathrm{C} 19-\mathrm{C} 2-\mathrm{C} 3$ & $109.35(18)$ & $\mathrm{C} 13-\mathrm{C} 12-\mathrm{H} 12$ & 119.0 \\
\hline $\mathrm{C} 18-\mathrm{C} 2-\mathrm{S} 1$ & $111.29(16)$ & $\mathrm{C} 11-\mathrm{C} 12-\mathrm{H} 12$ & 119.0 \\
\hline $\mathrm{C} 19-\mathrm{C} 2-\mathrm{S} 1$ & $105.39(15)$ & $\mathrm{C} 12-\mathrm{C} 13-\mathrm{C} 14$ & $120.26(19)$ \\
\hline $\mathrm{C} 3-\mathrm{C} 2-\mathrm{S} 1$ & $102.53(14)$ & $\mathrm{C} 12-\mathrm{C} 13-\mathrm{H} 13$ & 119.9 \\
\hline $\mathrm{C} 2-\mathrm{C} 3-\mathrm{C} 4$ & $119.60(18)$ & $\mathrm{C} 14-\mathrm{C} 13-\mathrm{H} 13$ & 119.9 \\
\hline $\mathrm{C} 2-\mathrm{C} 3-\mathrm{H} 3 \mathrm{~A}$ & 107.4 & $\mathrm{O} 1-\mathrm{C} 14-\mathrm{C} 15$ & $123.3(2)$ \\
\hline $\mathrm{C} 4-\mathrm{C} 3-\mathrm{H} 3 \mathrm{~A}$ & 107.4 & $\mathrm{O} 1-\mathrm{C} 14-\mathrm{C} 13$ & $117.9(2)$ \\
\hline $\mathrm{C} 2-\mathrm{C} 3-\mathrm{H} 3 \mathrm{~B}$ & 107.4 & $\mathrm{C} 15-\mathrm{C} 14-\mathrm{C} 13$ & $118.8(2)$ \\
\hline $\mathrm{C} 4-\mathrm{C} 3-\mathrm{H} 3 \mathrm{~B}$ & 107.4 & $\mathrm{C} 14-\mathrm{C} 15-\mathrm{C} 16$ & $120.2(2)$ \\
\hline $\mathrm{H} 3 \mathrm{~A}-\mathrm{C} 3-\mathrm{H} 3 \mathrm{~B}$ & 107.0 & $\mathrm{C} 14-\mathrm{C} 15-\mathrm{H} 15$ & 119.9 \\
\hline $\mathrm{C} 11-\mathrm{C} 4-\mathrm{C} 5$ & $108.25(16)$ & $\mathrm{C} 16-\mathrm{C} 15-\mathrm{H} 15$ & 119.9 \\
\hline $\mathrm{C} 11-\mathrm{C} 4-\mathrm{C} 17$ & $111.28(18)$ & $\mathrm{C} 11-\mathrm{C} 16-\mathrm{C} 15$ & $121.8(2)$ \\
\hline $\mathrm{C} 5-\mathrm{C} 4-\mathrm{C} 17$ & $108.20(17)$ & $\mathrm{C} 11-\mathrm{C} 16-\mathrm{H} 16$ & 119.1 \\
\hline $\mathrm{C} 11-\mathrm{C} 4-\mathrm{C} 3$ & $106.76(17)$ & $\mathrm{C} 15-\mathrm{C} 16-\mathrm{H} 16$ & 119.1 \\
\hline $\mathrm{C} 5-\mathrm{C} 4-\mathrm{C} 3$ & $113.09(18)$ & $\mathrm{C} 4-\mathrm{C} 17-\mathrm{H} 17 \mathrm{~A}$ & 109.5 \\
\hline $\mathrm{C} 17-\mathrm{C} 4-\mathrm{C} 3$ & $109.29(17)$ & $\mathrm{C} 4-\mathrm{C} 17-\mathrm{H} 17 \mathrm{~B}$ & 109.5 \\
\hline $\mathrm{C} 10-\mathrm{C} 5-\mathrm{C} 6$ & $116.23(19)$ & $\mathrm{H} 17 \mathrm{~A}-\mathrm{C} 17-\mathrm{H} 17 \mathrm{~B}$ & 109.5 \\
\hline $\mathrm{C} 10-\mathrm{C} 5-\mathrm{C} 4$ & $124.4(2)$ & $\mathrm{C} 4-\mathrm{C} 17-\mathrm{H} 17 \mathrm{C}$ & 109.5 \\
\hline $\mathrm{C} 6-\mathrm{C} 5-\mathrm{C} 4$ & $119.3(2)$ & $\mathrm{H} 17 \mathrm{~A}-\mathrm{C} 17-\mathrm{H} 17 \mathrm{C}$ & 109.5 \\
\hline $\mathrm{C} 7-\mathrm{C} 6-\mathrm{C} 5$ & $122.3(2)$ & $\mathrm{H} 17 \mathrm{~B}-\mathrm{C} 17-\mathrm{H} 17 \mathrm{C}$ & 109.5 \\
\hline $\mathrm{C} 7-\mathrm{C} 6-\mathrm{H} 6$ & 118.9 & $\mathrm{C} 2-\mathrm{C} 18-\mathrm{H} 18 \mathrm{~A}$ & 109.5 \\
\hline $\mathrm{C} 5-\mathrm{C} 6-\mathrm{H} 6$ & 118.9 & $\mathrm{C} 2-\mathrm{C} 18-\mathrm{H} 18 \mathrm{~B}$ & 109.5 \\
\hline $\mathrm{C} 6-\mathrm{C} 7-\mathrm{C} 8$ & $120.0(2)$ & $\mathrm{H} 18 \mathrm{~A}-\mathrm{C} 18-\mathrm{H} 18 \mathrm{~B}$ & 109.5 \\
\hline $\mathrm{C} 6-\mathrm{C} 7-\mathrm{H} 7$ & 120.0 & $\mathrm{C} 2-\mathrm{C} 18-\mathrm{H} 18 \mathrm{C}$ & 109.5 \\
\hline $\mathrm{C} 8-\mathrm{C} 7-\mathrm{H} 7$ & 120.0 & $\mathrm{H} 18 \mathrm{~A}-\mathrm{C} 18-\mathrm{H} 18 \mathrm{C}$ & 109.5 \\
\hline $\mathrm{C} 9-\mathrm{C} 8-\mathrm{C} 7$ & $119.2(2)$ & $\mathrm{H} 18 \mathrm{~B}-\mathrm{C} 18-\mathrm{H} 18 \mathrm{C}$ & 109.5 \\
\hline $\mathrm{C} 9-\mathrm{C} 8-\mathrm{H} 8$ & 120.4 & $\mathrm{C} 2-\mathrm{C} 19-\mathrm{H} 19 \mathrm{~A}$ & 109.5 \\
\hline $\mathrm{C} 7-\mathrm{C} 8-\mathrm{H} 8$ & 120.4 & $\mathrm{C} 2-\mathrm{C} 19-\mathrm{H} 19 \mathrm{~B}$ & 109.5 \\
\hline $\mathrm{C} 8-\mathrm{C} 9-\mathrm{C} 10$ & $120.0(2)$ & $\mathrm{H} 19 \mathrm{~A}-\mathrm{C} 19-\mathrm{H} 19 \mathrm{~B}$ & 109.5 \\
\hline $\mathrm{C} 8-\mathrm{C} 9-\mathrm{H} 9$ & 120.0 & $\mathrm{C} 2-\mathrm{C} 19-\mathrm{H} 19 \mathrm{C}$ & 109.5 \\
\hline $\mathrm{C} 10-\mathrm{C} 9-\mathrm{H} 9$ & 120.0 & $\mathrm{H} 19 \mathrm{~A}-\mathrm{C} 19-\mathrm{H} 19 \mathrm{C}$ & 109.5 \\
\hline $\mathrm{C} 9-\mathrm{C} 10-\mathrm{C} 5$ & $122.3(2)$ & $\mathrm{H} 19 \mathrm{~B}-\mathrm{C} 19-\mathrm{H} 19 \mathrm{C}$ & 109.5 \\
\hline
\end{tabular}




$\begin{array}{llll}\mathrm{O} 2-\mathrm{S} 1-\mathrm{C} 2-\mathrm{C} 18 & 45.94(19) & \mathrm{C} 6-\mathrm{C} 5-\mathrm{C} 10-\mathrm{C} 9 & 2.8(3) \\ \mathrm{C} 10-\mathrm{S} 1-\mathrm{C} 2-\mathrm{C} 18 & -64.08(18) & \mathrm{C} 4-\mathrm{C} 5-\mathrm{C} 10-\mathrm{C} 9 & -175.00(19) \\ \mathrm{O} 2-\mathrm{S} 1-\mathrm{C} 2-\mathrm{C} 19 & -73.49(17) & \mathrm{C} 6-\mathrm{C} 5-\mathrm{C} 10-\mathrm{S} 1 & -172.93(15) \\ \mathrm{C} 10-\mathrm{S} 1-\mathrm{C} 2-\mathrm{C} 19 & 176.50(15) & \mathrm{C} 4-\mathrm{C} 5-\mathrm{C} 10-\mathrm{S} 1 & 9.3(3) \\ \mathrm{O} 2-\mathrm{S} 1-\mathrm{C} 2-\mathrm{C} 3 & 172.10(14) & \mathrm{O} 2-\mathrm{S} 1-\mathrm{C} 10-\mathrm{C} 9 & 31.70(18) \\ \mathrm{C} 10-\mathrm{S} 1-\mathrm{C} 2-\mathrm{C} 3 & 62.09(15) & \mathrm{C} 2-\mathrm{S} 1-\mathrm{C} 10-\mathrm{C} 9 & 143.96(16) \\ \mathrm{C} 18-\mathrm{C} 2-\mathrm{C} 3-\mathrm{C} 4 & 54.9(3) & \mathrm{O} 2-\mathrm{S} 1-\mathrm{C} 10-\mathrm{C} 5 & -152.32(17) \\ \mathrm{C} 19-\mathrm{C} 2-\mathrm{C} 3-\mathrm{C} 4 & -178.82(18) & \mathrm{C} 2-\mathrm{S} 1-\mathrm{C} 10-\mathrm{C} 5 & -40.06(19) \\ \mathrm{S} 1-\mathrm{C} 2-\mathrm{C} 3-\mathrm{C} 4 & -67.3(2) & \mathrm{C} 5-\mathrm{C} 4-\mathrm{C} 11-\mathrm{C} 16 & -124.3(2) \\ \mathrm{C} 2-\mathrm{C} 3-\mathrm{C} 4-\mathrm{C} 11 & 152.40(17) & \mathrm{C} 17-\mathrm{C} 4-\mathrm{C} 11-\mathrm{C} 16 & -5.6(3) \\ \mathrm{C} 2-\mathrm{C} 3-\mathrm{C} 4-\mathrm{C} 5 & 33.5(2) & \mathrm{C} 3-\mathrm{C} 4-\mathrm{C} 11-\mathrm{C} 16 & 113.6(2) \\ \mathrm{C} 2-\mathrm{C} 3-\mathrm{C} 4-\mathrm{C} 17 & -87.1(2) & \mathrm{C} 5-\mathrm{C} 4-\mathrm{C} 11-\mathrm{C} 12 & 58.2(2) \\ \mathrm{C} 11-\mathrm{C} 4-\mathrm{C} 5-\mathrm{C} 10 & -117.8(2) & \mathrm{C} 17-\mathrm{C} 4-\mathrm{C} 11-\mathrm{C} 12 & 176.96(19) \\ \mathrm{C} 17-\mathrm{C} 4-\mathrm{C} 5-\mathrm{C} 10 & 121.5(2) & \mathrm{C} 3-\mathrm{C} 4-\mathrm{C} 11-\mathrm{C} 12 & -63.9(2) \\ \mathrm{C} 3-\mathrm{C} 4-\mathrm{C} 5-\mathrm{C} 10 & 0.3(3) & \mathrm{C} 16-\mathrm{C} 11-\mathrm{C} 12-\mathrm{C} 13 & 0.7(3) \\ \mathrm{C} 11-\mathrm{C} 4-\mathrm{C} 5-\mathrm{C} 6 & 64.5(2) & \mathrm{C} 4-\mathrm{C} 11-\mathrm{C} 12-\mathrm{C} 13 & 178.3(2) \\ \mathrm{C} 17-\mathrm{C} 4-\mathrm{C} 5-\mathrm{C} 6 & -56.2(2) & \mathrm{C} 11-\mathrm{C} 12-\mathrm{C} 13-\mathrm{C} 14 & -1.7(3) \\ \mathrm{C} 3-\mathrm{C} 4-\mathrm{C} 5-\mathrm{C} 6 & -177.41(19) & \mathrm{C} 12-\mathrm{C} 13-\mathrm{C} 14-\mathrm{O} 1 & -179.3(2) \\ \mathrm{C} 10-\mathrm{C} 5-\mathrm{C} 6-\mathrm{C} 7 & -1.7(3) & \mathrm{C} 12-\mathrm{C} 13-\mathrm{C} 14-\mathrm{C} 15 & 1.2(3) \\ \mathrm{C} 4-\mathrm{C} 5-\mathrm{C} 6-\mathrm{C} 7 & 176.2(2) & \mathrm{O} 1-\mathrm{C} 14-\mathrm{C} 15-\mathrm{C} 16 & -179.3(2) \\ \mathrm{C} 5-\mathrm{C} 6-\mathrm{C} 7-\mathrm{C} 8 & -0.6(4) & \mathrm{C} 13-\mathrm{C} 14-\mathrm{C} 15-\mathrm{C} 16 & 0.3(3) \\ \mathrm{C} 6-\mathrm{C} 7-\mathrm{C} 8-\mathrm{C} 9 & 2.0(4) & \mathrm{C} 12-\mathrm{C} 11-\mathrm{C} 16-\mathrm{C} 15 & 0.8(3) \\ \mathrm{C} 7-\mathrm{C} 8-\mathrm{C} 9-\mathrm{C} 10 & -1.0(3) & \mathrm{C} 4-\mathrm{C} 11-\mathrm{C} 16-\mathrm{C} 15 & -176.7(2) \\ \mathrm{C} 8-\mathrm{C} 9-\mathrm{C} 10-\mathrm{C} 5 & -1.5(3) & \mathrm{C} 14-\mathrm{C} 15-\mathrm{C} 16-\mathrm{C} 11 & -1.3(3) \\ \mathrm{C} 8-\mathrm{C} 9-\mathrm{C} 10-\mathrm{S} 1 & 174.49(17) & & \\ & & & \end{array}$

Hydrogen-bond geometry $\left(\AA,{ }^{\circ}\right)$

\begin{tabular}{lllll}
\hline$D-\mathrm{H} \cdots A$ & $D-\mathrm{H}$ & $\mathrm{H} \cdots A$ & $D \cdots A$ & $D-\mathrm{H} \cdots A$ \\
\hline $\mathrm{O} 1-\mathrm{H} 1 \cdots \mathrm{O} 2^{\mathrm{i}}$ & $0.77(3)$ & $1.92(3)$ & $2.685(3)$ & $169(3)$ \\
\hline
\end{tabular}

Symmetry code: (i) $x, y-1, z$. 